\title{
Generalising Bertrand competition: a special case of the Hotelling model
}

\author{
Manzur Rashid ${ }^{1}$
}

Received: 2 July 2014 / Accepted: 16 July 2015 / Published online: 28 July 2015

(C) The Author(s) 2015. This article is published with open access at Springerlink.com

\begin{abstract}
In the classic Bertrand duopoly, a firm need only undercut its competitor by an arbitrarily small amount in order to sell to all the consumers. This paper generalises the Bertrand model so that a firm must be more than $\varepsilon$ cheaper than its competitor in order to take the market. I characterise the unique symmetric mixed-strategy equilibrium. Firms earn strictly positive expected profits in equilibrium which are increasing in $\varepsilon$. The model is also a special case of the price-setting stage of the Hotelling model with a non-uniform distribution of consumers. The model can also be formulated as one of sequential search without replacement from a discrete distribution of prices.
\end{abstract}

Keywords Bertrand model · Discontinuous payoffs · Duopoly · Hotelling model · Price dispersion

JEL Classification L13 - D21 · D43

\section{Introduction}

In the classic Bertrand duopoly, firms split the market equally if and only if they offer exactly the same price. If one firm offers a price arbitrarily less than its competitor, it is able to sell to all of the consumers. This leads to fierce competition and marginal cost pricing in equilibrium. That firms make no profit in equilibrium is called the

I am grateful to Martin Cripps for his support and guidance. I am also grateful to Paola Manzini, Syngjoo Choi and two referees for detailed comments which have substantively improved the paper. This work was supported by the Economic and Social Research Council [Grant Number ES/I904166/1].

Manzur Rashid

manzur.rashid@ucl.ac.uk

1 Department of Management Science, UCL, Gower Street, London WC1E 6BT, UK 
Bertrand paradox: it seems strange that the model only requires one additional firm in the marketplace to go from the monopoly outcome to an outcome which simulates perfect competition. In this paper I provide a natural extension of the Bertrand model where firms split the market equally if their prices are sufficiently close to one another; there is a unique symmetric equilibrium where firms earn positive expected profits.

There are a number of papers which consider circumstances under which the Bertrand paradox disappears and firms earn positive profits in equilibrium. Baye and Morgan (1999) show that if the monopoly profit function is unbounded there exist positive profit equilibria. Dastidar (1995) shows that if firms have identical and strictly convex cost functions, positive profit equilibria exist even when the monopoly profit function is bounded. Baye and Morgan (2002) show that this result no longer holds under the 'winner-takes-all' tie-breaking rule. Baye and Kovenock (2008) show that with concave costs and a symmetric sharing rule, equilibria may not exist in either pure or mixed strategies. In my model, the monopoly profit function is bounded, firms have identical and constant marginal costs and the results are invariant to the choice of sharing rule. The only departure from the classic model is that the firms now tie if their prices are sufficiently close.

Another classic model of competition is due to Hotelling (1929) where firms initially choose their location and then compete on price. The equilibrium price distributions in my model are precisely the mixed strategies which would be chosen by firms in the price-setting stage of the Hotelling model for a restricted class of (non-uniform) distributions of consumers. ${ }^{1}$

Finally, my model has an extremely simple sequential search interpretation where two firms simultaneously choose a price. These two prices $\left\{p_{1}, p_{2}\right\}$ form a known discrete distribution from which consumers can take costly draws without replacement. In this respect, my model is closest to Stiglitz (1987) who models sequential search (both with and without replacement) from a known discrete distribution by consumers who differ in their search costs. However, he does not characterise the actual pricing strategies that firms use in equilibrium. Stahl (1989) does derive the equilibrium distribution of prices; however, in his model consumers do not know the realised distribution of prices, they only know the mixed strategies firms use in equilibrium. ${ }^{2}$ Furthermore, his model requires some consumers to have no search costs; otherwise, the equilibrium involves only monopoly pricing.

Ever since Diamond (1971) showed that even small search costs can lead to monopoly pricing, one of the key issues the literature has struggled with is explaining how price dispersion for homogeneous goods can be an equilibrium phenomenon. This is important since without price dispersion there is no need for consumers to search. A nice feature of Stahl's (1989) model is that as search costs increase, the dis-

\footnotetext{
1 There is a literature which deals with non-uniform distributions of consumers in the Hotelling model; see Anderson et al. (1997), Neven (1986), Tabuchi and Thisse (1995) and Shilony (1981).

2 Stahl (1996) stresses the distinction between models in which "consumers know the 'market distribution' of actual prices being charged but do not know which store is charging which price" and those models where consumers search optimally with respect to the mixed strategies firms use in equilibrium but without knowledge of the realisations (unless they search). My model is of the first type, others of this type include Salop and Stiglitz (1977), Braverman (1980), Rob (1985) and Stiglitz (1987). Models of the second type include Burdett and Judd (1983), Axell (1977) and Stahl (1989).
} 
tribution of prices smoothly increases from competitive to monopoly pricing-thus providing a bridge between the Bertrand paradox (zero profits) and the Diamond paradox (monopoly pricing). There is a similar effect in my model. Finally, in the unique equilibrium of my model, firms use mixed strategies, thus this extremely simple model is able to explain price dispersion without having to assume any heterogeneity of firms, consumers or goods. ${ }^{3}$

\section{The model}

There are two firms $(i=1,2)$ competing for a unit mass of consumers who demand one unit of a good which they value at $v=1$. Firms simultaneously choose a price. If the two prices are within $\varepsilon$ of each other, the firms split the market equally. If the two prices are greater than $\varepsilon$ apart, all the consumers purchase from the cheapest firm. Firms can produce the good costlessly. The firms have continuous action space $A_{i}=[0,1]$ and their profit function is given by:

$$
\pi_{i}\left(p_{i}, p_{j}\right)= \begin{cases}p_{i}, & p_{i}<p_{j}-\varepsilon \\ \frac{p_{i}}{2}, & \left|p_{i}-p_{j}\right| \leq \varepsilon \\ 0, & p_{i}>p_{j}+\varepsilon\end{cases}
$$

This fully specifies the game. We give three interpretations which give rise to the game: (1) the Bertrand interpretation (2) the Hotelling interpretation and (3) the search interpretation.

\section{The Bertrand interpretation}

Primarily, the model is a generalisation of the Bertrand model. In my model a firm must be sufficiently cheaper in order to attract all of the consumers. One reason for this might be that there is a small amount of heterogeneity in the goods being sold; half of the consumers have a preference for firm 1 while the other consumers have a preference for firm 2. Thus, in order to tempt consumers away from their competitor, a firm has to be at least $\varepsilon$ cheaper. Notice that the model with $\varepsilon=0$ collapses to Bertrand competition.

2. The Hotelling interpretation

In the standard Hotelling model, consumers are distributed uniformly. My model is a special case of the price-setting stage of the Hotelling model but with a nonuniform distribution of consumers. Letting $x_{i}$ be firm $i$ 's location on the unit interval (w.l.o.g. $x_{1} \leq x_{2}$ ). Suppose that transportation costs are linear (so that in order to travel from $a$ to $b$ it costs $|a-b|$ ) and the 'market is covered' so that all consumers value the good sufficiently highly to make a purchase in equilibrium. In this case, any distribution of consumers where half the consumers are located somewhere in the interval $\left[0, x_{1}\right]$ and the other half are located somewhere in the

\footnotetext{
3 Baye et al. (2007) provide an excellent review of models which attempt to explain price dispersion. Typical examples include Varian (1980): in his model it is necessary for some proportion of consumers to either not be optimising or have search costs which are so large that search is never optimal. Other papers introduce heterogeneity in firms' costs (e.g. Reinganum 1979) or consumers' search costs (e.g. Rob 1985). In Burdett and Judd (1983) consumers are able to search multiple firms in parallel.
} 
interval $\left[x_{2}, 1\right]$ gives rise to my model. The ' $\varepsilon$ ' in my model is then the distance between the two firms $x_{2}-x_{1}$.

If transportation costs are non-linear then the case where half the mass of consumers is located at $x_{1}$ and the other half are located at $x_{2}$ also gives rise to my model. Here, any increasing transportation cost function will do: what is important is that consumers agree on how preferable it is to purchase from the closer of the two firms.

3. The Search interpretation

The model can also be formulated as a simple sequential search model without replacement from a known discrete distribution of prices. Consider the following timing:

1. The two firms simultaneously choose a price each. ${ }^{4}$

2. Consumers know which two prices $\left\{p_{1}, p_{2}\right\}$ have been chosen, but they do not know which price is associated with which firm.

3. Consumers can search without replacement from the known distribution $\left\{p_{1}, p_{2}\right\}$ which contains only two discrete prices.

4. The first search is 'free' and at random: half of the consumers draw $p_{1}$ and the other half draw $p_{2}$.

5. Consumers can purchase immediately at the price they have drawn or they can pay $\varepsilon$ in order to take another draw (i.e. visit the other firm whose price is known and will be drawn for sure).

It is straightforward to see that if the two firms' prices are within $\varepsilon$ of one another, they split the market; while if one is more than $\varepsilon$ cheaper it sells to all of the consumers.

\subsection{Solving the model}

Here, I prove the substantive result, which is that there is a unique symmetric mixedstrategy equilibrium where firms offer prices according to the distribution

$$
F\left(p_{i}\right)= \begin{cases}1-\frac{(1+\sqrt{2}) \varepsilon}{p_{i}+\varepsilon}, & p_{i} \in[\sqrt{2} \varepsilon,(1+\sqrt{2}) \varepsilon] \\ 2-\frac{(1+\sqrt{2}) \varepsilon}{p_{i}-\varepsilon}, & p_{i} \in[(1+\sqrt{2}) \varepsilon,(2+\sqrt{2}) \varepsilon] .\end{cases}
$$

I begin by using dominance arguments in order to simplify the problem.

Lemma 1 Prices in the interval $[0, \sqrt{2} \varepsilon$ ) do not survive the iterated deletion of prices which are strictly dominated.

Proof If firm $i$ offers a price of $\varepsilon$ and firm $j$ offers a price $p_{j}$, profits to firm $i$ will be

$$
\pi_{i}\left(\varepsilon, p_{j}\right)= \begin{cases}\frac{\varepsilon}{2}, & p_{j} \in[0,2 \varepsilon] \\ \varepsilon, & p_{j}>2 \varepsilon\end{cases}
$$

By offering the price $\varepsilon$, firm $i$ can guarantee itself at least a payoff of $\varepsilon / 2$. Thus, offering a price less than $\varepsilon / 2$ must be strictly dominated by offering the price $\varepsilon$. Now consider

4 Firms are allowed to randomise, but a single price must be chosen by each firm here. 


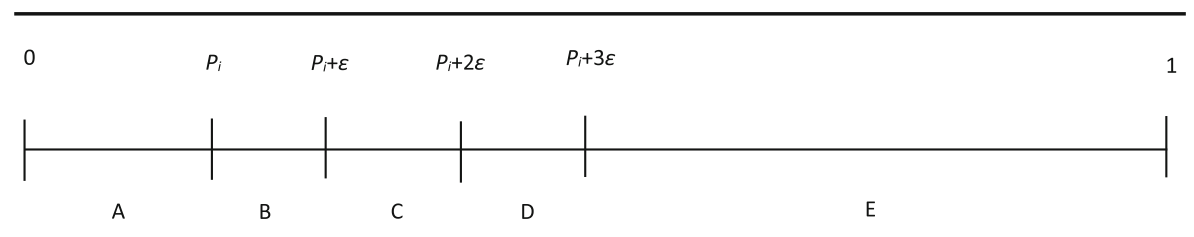

Fig. 1 Partitioning the action space into regions

firm $i$ offering the price $3 \varepsilon / 2$, depending on $p_{j}$, this will yield profits

$$
\pi_{i}\left(3 \varepsilon / 2, p_{j}\right)=\left\{\begin{array}{l}
\frac{3 \varepsilon}{4}, p_{j} \in\left[\frac{\varepsilon}{2}, \frac{5 \varepsilon}{2}\right] \\
\frac{3 \varepsilon}{2}, p_{j}>\frac{5 \varepsilon}{2}
\end{array}\right.
$$

Since no firm will offer a price less than $\varepsilon / 2$, firm $i$ can guarantee itself a payoff of at least $3 \varepsilon / 4$. This allows us to delete prices less than $3 \varepsilon / 4$. Iterating in this fashion allows us to delete all prices less than $\varepsilon$. We can in fact delete more prices than this. Consider the pure strategy $s_{i}$ which plays $p_{i} \geq \varepsilon$ for sure, and the alternative strategy $s_{i}^{\prime}$ which involves playing $p_{i}+\varepsilon$ with probability $\sigma$ and $p_{i}+2 \varepsilon \leq 1$ with probability $1-\sigma$ (Fig. 1).

Consider profits to firm $i$-when playing the two different strategies—when firm $j$ prices in the different regions:

Region A: Since we are searching for the limit of iteratively deleting dominated strategies, we need not consider firm $j$ pricing in this interval since it would have been deleted in some earlier round.

Region B: $\pi_{i}\left(s_{i}, B\right)=\frac{1}{2} p_{i}, \pi_{i}\left(s_{i}^{\prime}, B\right)=\frac{\sigma}{2}\left(p_{i}+\varepsilon\right)$.

In order for strategy $s_{i}^{\prime}$ to strictly dominate strategy $s_{i}$, it must be that

$$
\pi_{i}\left(s_{i}^{\prime}, B\right)>\pi_{i}\left(s_{i}, B\right) \Leftrightarrow \sigma>\frac{p_{i}}{p_{i}+\varepsilon} .
$$

Region C: $\pi_{i}\left(s_{i}, C\right)=p_{i}, \pi_{i}\left(s_{i}^{\prime}, C\right)=\frac{1}{2}\left[\sigma\left(p_{i}+\varepsilon\right)+(1-\sigma)\left(p_{i}+2 \varepsilon\right)\right]$.

In order for strategy $s_{i}^{\prime}$ to strictly dominate strategy $s_{i}$, it must be that

$$
\pi_{i}\left(s_{i}^{\prime}, C\right)>\pi_{i}\left(s_{i}, C\right) \Leftrightarrow \frac{1}{2}\left[\sigma\left(p_{i}+\varepsilon\right)+(1-\sigma)\left(p_{i}+2 \varepsilon\right)\right]>p_{i} .
$$

Call $\hat{p}$ the lowest price which cannot be shown to be strictly dominated using this argument. If this is the case, $\hat{p}$ will (just) fail to satisfy the conditions (1) and (2), so that

$$
\sigma=\frac{\hat{p}}{\hat{p}+\varepsilon} \quad \text { and } \quad \frac{1}{2}[\sigma(\hat{p}+\varepsilon)+(1-\sigma)(\hat{p}+2 \varepsilon)]=\hat{p}
$$

Solving for $\hat{p}$ give us that $\hat{p}=\sqrt{2} \varepsilon$.

Region D: $\pi_{i}\left(s_{i}, D\right)=p_{i}, \pi_{i}\left(s_{i}^{\prime}, D\right)=\sigma\left(p_{i}+\varepsilon\right)+\frac{1-\sigma}{2}\left(p_{i}+2 \varepsilon\right)$. 


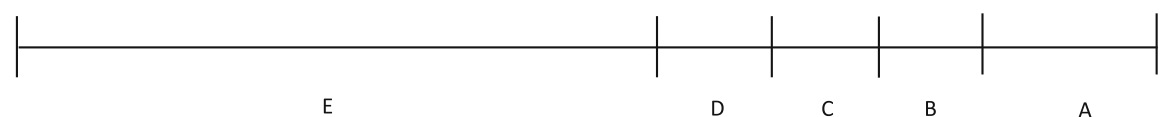

Fig. 2 Partitioning the action space into regions

In order for strategy $s_{i}^{\prime}$ to strictly dominate strategy $s_{i}$, it must be that

$$
\pi_{i}\left(s_{i}^{\prime}, D\right)>\pi_{i}\left(s_{i}, D\right) \Leftrightarrow \sigma\left(p_{i}+\varepsilon\right)+\frac{1-\sigma}{2}\left(p_{i}+2 \varepsilon\right)>p_{i} .
$$

This condition will always be satisfied if (2) is satisfied.

Region E: Here both strategies attract all the consumers, meaning that $s_{i}^{\prime}$ must do strictly better than $s_{i}$ since it is associated with strictly higher prices.

Lemma 2 Prices in the interval $(4 \varepsilon, 1]$ do not survive the iterated deletion of prices which are strictly dominated.

Proof Now consider which prices we can iteratively delete from the top of the interval $[0,1]$. Again $s_{i}$ is a pure strategy which plays $p_{i}$ for sure, while $s_{i}^{\prime}$ this time, plays $p_{i}-2 \varepsilon$ for sure (Fig. 2).

Consider profits to firm $i$-when playing the two different strategies-when firm $j$ prices in the different regions:

Region A: Prices in this region will have been deleted in some earlier round of deletion.

Region B: $\pi_{i}\left(s_{i}, B\right)=\frac{1}{2} p_{i}, \pi_{i}\left(s_{i}^{\prime}, B\right)=p_{i}-2 \varepsilon$.

In order for strategy $s_{i}^{\prime}$ to strictly dominate strategy $s_{i}$, it must be that

$$
\pi_{i}\left(s_{i}^{\prime}, B\right)>\pi_{i}\left(s_{i}, B\right) \Leftrightarrow p_{i}>4 \varepsilon .
$$

Region C and D: $\pi_{i}\left(s_{i}, C\right)=\pi_{i}\left(s_{i}, D\right)=0, \pi_{i}\left(s_{i}^{\prime}, C\right)=\pi_{i}\left(s_{i}^{\prime}, D\right)=\frac{1}{2}\left(p_{i}-2 \varepsilon\right)$.

If condition (4) holds, strategy $s_{i}^{\prime}$ does strictly better than strategy $s_{i}$ here. Region $\mathrm{E}: \pi_{i}\left(s_{i}, E\right)=\pi_{i}\left(s_{i}^{\prime}, E\right)=0$.

Here, $s_{i}^{\prime}$ does not do strictly better than $s_{i}$. However, we will still be okay if we amend strategy $s_{i}^{\prime}$ (call it $s_{i}^{\prime \prime}$ ) so that instead of offering the price $p_{i}-2 \varepsilon$ for sure, it offers it with probability $1-\mu$. Where $\mu$ is some small strictly positive real number. With the remaining $\mu$ probability, strategy $s_{i}^{\prime \prime}$ is equally likely to offer one of the prices $p_{i}-4 \varepsilon, p_{i}-6 \varepsilon, p_{i}-8 \varepsilon$ and so on. This will mean that $\pi_{i}\left(s_{i}^{\prime \prime}, E\right)>0$. Furthermore, strategy $s_{i}^{\prime \prime}$ does almost as well as strategy $s_{i}^{\prime}$ in the other regions. Formally, for any $p_{i}>$ $4 \varepsilon$, one can always find a $\mu>0$ sufficiently small such that $\pi_{i}\left(s_{i}^{\prime \prime}, B\right)>\pi_{i}\left(s_{i}, B\right)$. In the other regions, it is easy to see that $\pi_{i}\left(s_{i}^{\prime \prime}\right)>\pi_{i}\left(s_{i}\right)$ for sufficiently small $\mu$.

We know that any candidate for an equilibrium must only offer prices in the interval $[\sqrt{2} \varepsilon, 4 \varepsilon]$ with positive probability. We now argue that there can be no mass points in the equilibrium strategy. 


\section{Lemma 3 There can be no mass points in the equilibrium strategy.}

Proof Suppose that there was a mass point in the equilibrium strategy of firm $i$ at price $p$. Firm $j$ reasons that there will be a discontinuous fall in its profit at $p+\varepsilon$. This is because at a price of $p+\varepsilon$ firm $j$ will 'draw' with the mass (i.e. they will split the market equally), but at a price above $p+\varepsilon$, firm $j$ will 'lose' against the mass. Thus, for firm $j$, playing the price $p+\varepsilon$ dominates the prices in some interval $(p+\varepsilon, q]$. Firm $j$ also notices that there is a discontinuous rise in profit at a price less than $p-\varepsilon$ which causes some interval $[p-\varepsilon, r]$ to be dominated. This means that firm $j$ will not play in either of these intervals if there is a mass at $p$. Firm $i$ now notices that there exists some price greater than $p$ which attracts the same proportion of consumers as $p$, thus there can be no mass at $p$.

Since there are no mass points, it follows that a symmetric equilibrium, if it exists, must involve firms offering prices in a single continuous interval or a number of continuous intervals. We will prove that it cannot be that there are a number of continuous intervals, there must be only one. First we will need another lemma.

Lemma 4 The support of the symmetric equilibrium distribution of prices $F$ can be partitioned into pairs of intervals which are $\varepsilon$ apart. Furthermore, if the pair $([a, b],[a+\varepsilon, b+\varepsilon])$ is in the support then it cannot be that prices in the interval $[a-\varepsilon, b-\varepsilon]$ or the interval $[a+2 \varepsilon, b+2 \varepsilon]$ are in the support of $F$.

Proof The proof is in two parts.

1. If the interval $[x, y]$ is in the support of $F$ it must be that we can partition it so that for every partitioned subinterval $[a, b]$ there is at least one corresponding interval $[a-\varepsilon, b-\varepsilon]$ and/or $[a+\varepsilon, b+\varepsilon]$ which is also in the support of $F$.

2. It cannot be that all three intervals $([a-\varepsilon, b-\varepsilon],[a, b],[a+\varepsilon, b+\varepsilon])$ are played in equilibrium.

1. In equilibrium, profits in the interval $[a, b]$ must be constant, thus a firm must be indifferent between charging a price $p \in(a, b]$ and a price slightly below $p$, say $p^{\prime} \in[a, b)$. In order to compensate the firm for the lower price, at the price $p^{\prime}$ it must be that they (in expectation) attract some additional consumers. This is only possible when their opponent offers prices in the interval $\left[p^{\prime}-\varepsilon, p-\varepsilon\right]$ and/or the interval $\left[p^{\prime}+\varepsilon, p+\varepsilon\right]$.

2. Suppose that all three intervals are played in equilibrium. Suppose firm $j$ randomises according to $F$. If firm $i$ chooses a price $p_{i} \in[a-\varepsilon, b-\varepsilon]$ this will yield an expected profit of:

$$
E\left[\pi_{i}\left(p_{i}, F\right)\right]=\left[1-\frac{1}{2} F\left(p_{i}+\varepsilon\right)\right] p_{i} .
$$

From Lemmas 1 and 2 we know that at these prices, firm $i$ will sell to either, half of the consumers or all of the consumers, i.e. it is not possible for firm $j$ to offer a price less than $b-2 \varepsilon$ and sell to the entire market as this would mean that the range of prices being offered would be at least $3 \varepsilon$. Since $E\left[\pi_{i}\left(p_{i}, F\right)\right]$ must be 
constant for all prices in the support, we can let $E\left[\pi_{i}\left(p_{i}, F\right)\right]=\pi$ and rearrange the above to get

$$
F\left(p_{i}+\varepsilon\right)=2\left[1-\frac{\pi}{p_{i}}\right], \quad p_{i} \in[a-\varepsilon, b-\varepsilon] .
$$

This tells us about the behaviour of $F$ for prices in the interval $[a, b]$. Yielding

$$
F\left(p_{i}\right)=2\left[1-\frac{\pi}{p_{i}-\varepsilon}\right], \quad p_{i} \in[a, b] .
$$

Similarly, if firm $i$ chooses a price $p_{i} \in[a+\varepsilon, b+\varepsilon]$ this will yield an expected profit of

$$
E\left[\pi_{i}\left(p_{i}, F\right)\right]=\frac{1}{2}\left(1-F\left(p_{i}-\varepsilon\right)\right) p_{i}
$$

Again, setting $E\left[\pi_{i}\left(p_{i}, F\right)\right]=\pi$ and rearranging we have

$$
F\left(p_{i}-\varepsilon\right)=1-\frac{2 \pi}{p_{i}}, \quad p_{i} \in[a+\varepsilon, b+\varepsilon] .
$$

This tells us about the behaviour of $\mathrm{F}$ for prices in the interval $[a, b]$. Yielding

$$
F\left(p_{i}\right)=1-\frac{2 \pi}{p_{i}+\varepsilon}, \quad p_{i} \in[a, b]
$$

We now have two expressions that $F$ must satisfy in order to qualify as a candidate for an equilibrium. We need that they are consistent with one another, which requires

$$
\begin{aligned}
& 2\left[1-\frac{\pi}{p_{i}-\varepsilon}\right]=1-\frac{2 \pi}{p_{i}+\varepsilon}, \quad p_{i} \in[a, b] \\
& \Rightarrow p_{i}=\left[4 \pi \varepsilon+\varepsilon^{2}\right]^{\frac{1}{2}} \quad p_{i} \in[a, b] .
\end{aligned}
$$

This cannot be true for any range of prices since this tells us that $p_{i}$ is equal to a constant.

We can now describe the exact interval of prices which must be played in any symmetric equilibrium.

Lemma 5 The support of the symmetric equilibrium distribution-if it exists-must be one continuous interval $[\sqrt{2} \varepsilon,(2+\sqrt{2}) \varepsilon]$. 
Proof We know from Lemma 4 that intervals of prices must occur in pairs in the equilibrium price distribution. ${ }^{5}$ Consider a distribution $F$, which consists only of playing the distinct intervals $\left[a_{0}, b_{0}\right],\left[a_{1}, b_{1}\right], \ldots,\left[a_{n}, b_{n}\right],\left[a_{0}+\varepsilon, b_{0}+\varepsilon\right], \ldots,\left[a_{n}+\right.$ $\left.\varepsilon, b_{n}+\varepsilon\right]$. We can show that in order for this to be a candidate for a symmetric equilibrium it must be that there are no gaps in between these intervals. From the proof of Lemma 4 we know that for each pair, the 'lower' interval, i.e. the intervals $\left[a_{0}, b_{0}\right],\left[a_{1}, b_{1}\right], \ldots,\left[a_{n}, b_{n}\right]$ must have CDF

$$
F\left(p_{i}\right)=1-\frac{2 \pi}{p_{i}+\varepsilon} .
$$

While the 'upper' interval, i.e. the intervals $\left[a_{0}+\varepsilon, b_{0}+\varepsilon\right], \ldots,\left[a_{n}+\varepsilon, b_{n}+\varepsilon\right]$ must have $\mathrm{CDF}$

$$
F\left(p_{i}\right)=2\left[1-\frac{\pi}{p_{i}-\varepsilon}\right]
$$

Since our distribution is atomless it must be that $F\left(a_{0}\right)=0 \Rightarrow \pi=\frac{1}{2}\left(a_{0}+\varepsilon\right)$ and also $F\left(b_{n}+\varepsilon\right)=1 \Rightarrow \pi=\frac{b_{n}}{2}$. Equating these two expressions for profits tells us

$$
\frac{1}{2}(a+\varepsilon)=\frac{b_{n}}{2} \Rightarrow b_{n}=a_{0}+\varepsilon .
$$

So, if the smallest price being played is $a_{0}$, the largest prices being played must be $a_{0}+2 \varepsilon$. Again using the fact that the distribution is atomless it must be that $F\left(b_{k}\right)=F\left(a_{k+1}\right) ; k=0,1, \ldots, n-1$. Which, in conjunction with our knowledge of the CDF, implies that $b_{k}=a_{k+1} ; k=0,1, \ldots, n-1$. This tells us that there will be no gaps in the distribution between $\left[a_{0}, b_{\mathrm{n}}\right]$.

Inspecting the upper intervals we see that it must be that $F\left(b_{k}+\varepsilon\right)=$ $F\left(a_{k+1}+\varepsilon\right) ; k=0,1, \ldots, n-1$. Which implies that $b_{k}+\varepsilon=a_{k+1}+\varepsilon ; k=$ $0,1, \ldots, n-1$. This tells us that there will be no gaps in the distribution between $\left[a_{0}+\varepsilon, b_{\mathrm{n}}+\varepsilon\right]$. Finally, we need to check that these two intervals join together continuously at $a_{0}+\varepsilon$. That is, we need

$$
1-\left.\frac{2 \pi}{p_{i}+\varepsilon}\right|_{p_{i}=a_{0}+\varepsilon}=\left.2\left[1-\frac{\pi}{p_{i}-\varepsilon}\right]\right|_{p_{i}=a_{0}+\varepsilon} .
$$

Using $\pi=\frac{1}{2}\left(a_{0}+\varepsilon\right)$ and evaluating give us

$$
\frac{\varepsilon}{a_{0}+2 \varepsilon}=\frac{a_{0}-\varepsilon}{a_{0}} \Rightarrow a_{0}=\sqrt{2} \varepsilon
$$

We can now state and prove the substantive result.

5 A pair being two intervals $[x, y]$ and $[x+\varepsilon, y+\varepsilon]$. 
Result There is a unique symmetric mixed-strategy Nash equilibrium where both firms offer prices according to: ${ }^{6}$

$$
F\left(p_{i}\right)= \begin{cases}1-\frac{(1+\sqrt{2}) \varepsilon}{p_{i}+\varepsilon}, & p_{i} \in[\sqrt{2} \varepsilon,(1+\sqrt{2}) \varepsilon] \\ 2-\frac{(1+\sqrt{2}) \varepsilon}{p_{i}-\varepsilon}, & p_{i} \in[(1+\sqrt{2}) \varepsilon,(2+\sqrt{2}) \varepsilon] .\end{cases}
$$

Expected profits to each firm are $\pi=\frac{1}{2}(1+\sqrt{2}) \varepsilon$.

Proof We know from Lemma 5 that our candidate for a symmetric equilibrium must satisfy

$$
F\left(p_{i}\right)= \begin{cases}1-\frac{2 \pi}{p_{i}+\varepsilon}, & p_{i} \in[\sqrt{2} \varepsilon,(1+\sqrt{2}) \varepsilon] ; \\ 2-\frac{2 \pi}{p_{i}-\varepsilon}, & p_{i} \in[(1+\sqrt{2}) \varepsilon,(2+\sqrt{2}) \varepsilon] .\end{cases}
$$

Since the support is of $2 \varepsilon$ length, offering the price exactly in the centre of the support will guarantee that a firm receives exactly half of the consumers. This tells us that expected profit must be $\pi=\frac{1}{2}(1+\sqrt{2}) \varepsilon$, plugging this into $F$ yields our result.

Finally, we need to check that a firm cannot do better by pricing outside of the interval when its opponent is offering prices according to $F$. We need to check two possible intervals of prices: $(1)[(2+\sqrt{2}) \varepsilon,(3+\sqrt{2}) \varepsilon]$ and $(2)[(-1+\sqrt{2}) \varepsilon, \sqrt{2} \varepsilon]$. This is because at prices greater than $(3+\sqrt{2}) \varepsilon$ firms will attract no customers and a price of $(-1+\sqrt{2}) \varepsilon$ is sufficiently low to attract all consumers. Pricing in interval (1) yields

$$
\begin{aligned}
& E\left[\pi_{i}\left(p_{i}, F\right)\right]=\left[1-F\left(p_{i}-\varepsilon\right)\right] \frac{p_{i}}{2} \\
& \Rightarrow \pi_{i}^{\prime}\left(p_{i}\right)=\frac{(1+\sqrt{2}) \varepsilon}{p_{i}-2 \varepsilon}\left[1-\frac{p_{i}}{p_{i}-2 \varepsilon}\right]-1<0 .
\end{aligned}
$$

Thus $p_{i}=(2+\sqrt{2}) \varepsilon$ must do strictly better than all other prices in this interval. Pricing in interval (2) yields

$$
\begin{aligned}
& E\left[\pi_{i}\left(p_{i}, F\right)\right]=\left[1-\frac{1}{2} F\left(p_{i}+\varepsilon\right)\right] p_{i} \\
& \Rightarrow \pi_{i}^{\prime}\left(p_{i}\right)=\frac{1}{2}+\frac{(1+\sqrt{2}) \varepsilon}{2\left(p_{i}+2 \varepsilon\right)}\left[1-\frac{p_{i}}{p_{i}+2 \varepsilon}\right]>0 .
\end{aligned}
$$

Thus $p_{i}=\sqrt{2} \varepsilon$ must do strictly better than all other prices in this interval.

\footnotetext{
6 To ensure existence we need that $\varepsilon$ is not too large compared to consumers' valuations, in particular $\varepsilon \leq 1 /(2+\sqrt{2})$. This ensures that prices offered are contained within the interval $[0,1]$.
} 


\section{Concluding remarks}

The simple model in this paper makes a contribution to the literature in three areas of economic theory: Bertrand competition, the Hotelling model and search theory.

Given the canonical status of the Bertrand model it is surprising that (to my knowledge) no paper has attempted to extend the model in the direction presented here. We are essentially answering the question: what happens when there exists some kind of friction in the marketplace which means that consumers only switch between firms if prices are sufficiently different? We have remained agnostic regarding the source of this friction but it could include switching costs, product differentiation, search costs, transportation costs or some other kind of consumer inertia.

The model generalises the Bertrand model of price competition and in doing so moves away from the Bertrand paradox of competitive pricing and zero profits in equilibrium. At the same time, for small frictions, prices in equilibrium are close to the competitive benchmark. As frictions increase, prices become, on average, both higher and more dispersed. For sufficiently large frictions, monopoly pricing results. ${ }^{7}$ That is, the model provides a bridge between competitive pricing (the Bertrand paradox) and monopoly pricing (the Diamond paradox). The model also provides an analytical solution to the equilibrium pricing strategies firms use in the price-setting stage of the Hotelling model with arbitrary locations, albeit with (very) restricted distributions of consumers.

Finally, as a model of sequential search, the model is limited by only being able to solve for the two-firm case. Nevertheless, the model can explain price dispersion in a simple optimising model without having to assume any heterogeneity of consumers, firms or goods. As far as I am aware, there is no other paper where consumers search sequentially for a single good where this is the case. A natural extension would be to solve the model with $N$ firms where consumers search sequentially without replacement from $N$ discrete prices. ${ }^{8}$

Open Access This article is distributed under the terms of the Creative Commons Attribution 4.0 International License (http://creativecommons.org/licenses/by/4.0/), which permits unrestricted use, distribution, and reproduction in any medium, provided you give appropriate credit to the original author(s) and the source, provide a link to the Creative Commons license, and indicate if changes were made.

\section{References}

Anderson, S.P., Goeree, J.K., Ramer, R.: Location, location, location. J. Econ. Theory 77, 102-127 (1997) Axell, B.: Search market equilibrium. Scand. J. Econ. 79, 20-40 (1977)

\footnotetext{
7 This is the case for $\varepsilon \leq 1 / 2$. To see this, notice that if both firms offer the monopoly price of 1 , they each make a profit of $1 / 2$. In order to sell to the entire market a price of less than $1-\varepsilon$ will have to be offered. For $\varepsilon \leq 1 / 2$, this is not a profitable deviation.

8 Although I am unable to solve for the equilibrium with $N$ firms, as the number of firms increase, competitive forces in the model decline. This is because it becomes more difficult for any individual firm to 'induce search' by offering a low price. I am able to characterise the minimum search cost required for monopoly pricing to be an equilibrium when there are $N$ firms and show that this is decreasing in $N$. As $N \rightarrow \infty$, monopoly pricing is an equilibrium for any strictly positive $\varepsilon$, thus reinstating the Diamond paradox.
} 
Baye, M.R., Kovenock, D.: Bertrand competition. In: Durlauf, S.N., Blume, L.E. (eds.) The New Palgrave Dictionary of Economics. Palgrave Macmillan, Basingstoke (2008)

Baye, M.R., Morgan, J.: A folk theorem for one-shot Bertrand games. Econ. Lett. 65, 59-65 (1999)

Baye, M.R., Morgan, J.: Winner-take-all price competition. Econ. Theory 19, 271-282 (2002)

Baye, M.R., Morgan, J., Scholten, P.: Information, search, and price dispersion. In: Hendershott, T. (ed.) Handbook of Economics and Systems. North-Holland, Amsterdam (2007)

Braverman, A.: Consumer search and alternative market equilibria. Rev. Econ. Stud. 47, 487-502 (1980)

Burdett, K., Judd, K.: Equilibrium price dispersion. Econometrica 51, 955-969 (1983)

Dastidar, K.G.: On the existence of pure strategy Bertrand equilibrium. Econ. Theory 5, 19-32 (1995)

Diamond, P.: A model of price adjustment. J. Econ. Theory 3, 156-168 (1971)

Hotelling, H.: Stability in competition. Econ. J. 39, 41-57 (1929)

Neven, D.: On Hotelling's competition with non-uniform consumer distributions. Econ. Lett. 21, 121-126 (1986)

Rob, R.: Equilibrium price distributions. Rev. Econ. Stud. 52, 457-504 (1985)

Reinganum, J.F.: A simple model of equilibrium price dispersion. J. Polit. Econ. 87, 851-858 (1979)

Salop, S., Stiglitz, J.: Bargains and ripoffs: a model of monopolistically competitive price dispersion. Rev. Econ. Stud. 44, 493-510 (1977)

Shilony, Y.: Hotelling's competition with general customer distributions. Econ. Lett. 8, 39-45 (1981)

Stahl, D.O.: Oligopolistic pricing with sequential consumer search. Am. Econ. Rev. 79, 700-712 (1989)

Stahl, D.O.: Oligopolistic pricing with heterogeneous consumer search. Int. J. Ind. Org. 14, 243-268 (1996)

Stiglitz, J.: Competition and the number of firms in a market: are duopolies more competitive than atomistic markets? J. Polit. Econ. 95, 1041-1061 (1987)

Tabuchi, T., Thisse, J.-F.: Asymmetric equilibria in spatial competition. Int. J. Ind. Org. 13, $213-227$ (1995)

Varian, H.R.: A model of sales. Am. Econ. Rev. 70, 651-659 (1980) 